\title{
Wooden Geometric Puzzles: Design and Hardness Proofs
}

\author{
Helmut Alt • Hans Bodlaender • \\ Marc van Kreveld • Günter Rote • Gerard Tel
}

Published online: 4 March 2008

(C) The Author(s) 2008. This article is published with open access at Springerlink.com

\begin{abstract}
We discuss some new geometric puzzles and the complexity of their extension to arbitrary sizes. For gate puzzles and two-layer puzzles we prove NP-completeness of solving them. Not only the solution of puzzles leads to interesting questions, but also puzzle design gives rise to interesting theoretical questions. This leads to the search for instances of partition that use only integers and are uniquely solvable. We show that instances of polynomial size exist with this property. This result also holds for partition into $k$ subsets with the same sum: We construct instances of $n$ integers with subset sum $O\left(n^{k+1}\right)$, for fixed $k$.
\end{abstract}

Keywords Geometric puzzles · Complexity · Partition

Part of this research was done when the first author was visiting Utrecht University, and during the Dagstuhl Seminar No. 06481 on Geometric Networks and Metric Space Embeddings in 2006.

H. Alt · G. Rote

Department of Computer Science, Freie Universität Berlin, Berlin, Germany

H. Alt

e-mail: alt@inf.fu-berlin.de

G. Rote

e-mail: rote@inf.fu-berlin.de

H. Bodlaender · M. van Kreveld $(\bowtie) \cdot$ G. Tel

Department of Information and Computing Sciences, Utrecht University, Utrecht, Netherlands e-mail:marc@cs.uu.nl

H. Bodlaender

e-mail: hansb@cs.uu.nl

G. Tel

e-mail: gerard@cs.uu.nl 


\section{Introduction}

Many good puzzles are instances of problems that are in general NP-complete. Conversely, NP-complete problems may be the inspiration for the design of nice puzzles. This is true for puzzles based on combinatorics, graphs, and geometry.

A puzzler's classification system of geometric puzzles exists that includes the classes Put-Together, Take Apart, Sequential Movement, and various others [1]. Although instances of puzzles in these classes have constant size, the natural generalization of many of them to sizes based on some parameter are NP-complete. For example, Instant Insanity is NP-complete [7, 10], sliding block puzzles like the 15-puzzle, Sokoban, and Rush Hour are NP-complete or PSPACE-complete [2, 6, 9], and puzzles related to packing like Tetris are NP-complete [5]. Some overviews are given by Demaine [3] and Demaine and Demaine [4].

In this paper we discuss some new geometric puzzles of the Put-Together type and analyze their complexity. We also discuss the creation of good instances of certain geometric puzzles based on set partition.

Gate Puzzles Gate puzzles consist of a board that is a regular square grid of holes and number of pieces called gates. Gates consist of three rods, two vertical and one horizontal, connecting the tops of the vertical rods. The vertical rods are called legs and have a certain leg distance that allows the gate to be placed on the board. A gate has a leg distance of 1 if the two legs are in adjacent holes. Furthermore, gates have a height, taken from a small set of values. To solve a gate puzzle, a given set of gates must be placed in the board. Every hole of the board must contain exactly one of the legs, and two gates can only intersect in the vertical projection if they have a different height, and the intersection is not at the vertical rods of the higher gate. Figure 1 shows an example. On the left, a $5 \times 5$ grid is shown with eleven normal gates of heights 2, 3, and 4, and three loose pegs (one-legged gates) of height 1 (which are
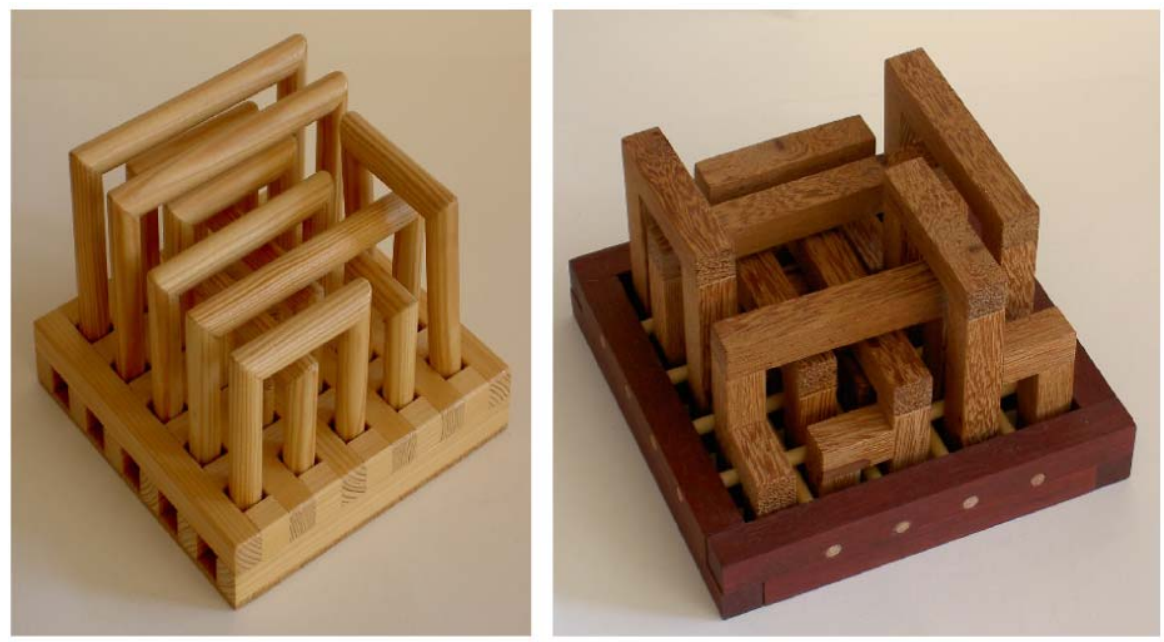

Fig. 1 Gate puzzles. Left with two-legged gates, right also with three-legged gates 
below the gates and not visible in the figure). On the right, a variation is shown where many gates have an extra leg: Two gates have two legs and seven gates have three legs. Most puzzlers take half an hour to a full hour to solve one of these puzzles. Gate puzzles were first described by the third author in [12]. In this paper we show that solving gate puzzles is NP-complete, which we prove by reduction from the strongly NP-complete problem 3-PARTITION (see for instance [7]).

Two-Layer Puzzles Two-layer puzzles consist of a set of pieces that must be arranged in two layers, where touching pieces from opposing layers must fit. The simplest type of such a puzzle consists of $2 k$ pieces of base $k \times 1$, and every $1 \times 1$ unit has a height 1 or 2 . The pieces must be arranged to make a solid $k \times k \times 3$ block. To this end, $k$ of the pieces must be arranged as rows, and the other $k$ pieces must be arranged upside down and as columns. Other two-layer puzzles can have pieces that use more than two heights, or pieces that do not have different heights, but use slanted tops in one of the four orientations [11]. See Fig. 2 for two examples.

A different realization of simplest type of two-layer puzzle is also known as the 16-holes puzzle. It consists of eight flat pieces of $4 \times 1$, with one, two or three holes. The objective is to cover the 16 holes of a $4 \times 4$ grid by placing the pieces on the grid in two layers, see Fig. 3.

Two-layer puzzles are NP-complete to solve, which we prove by reduction from Hamiltonian Circuit on graphs of degree three.
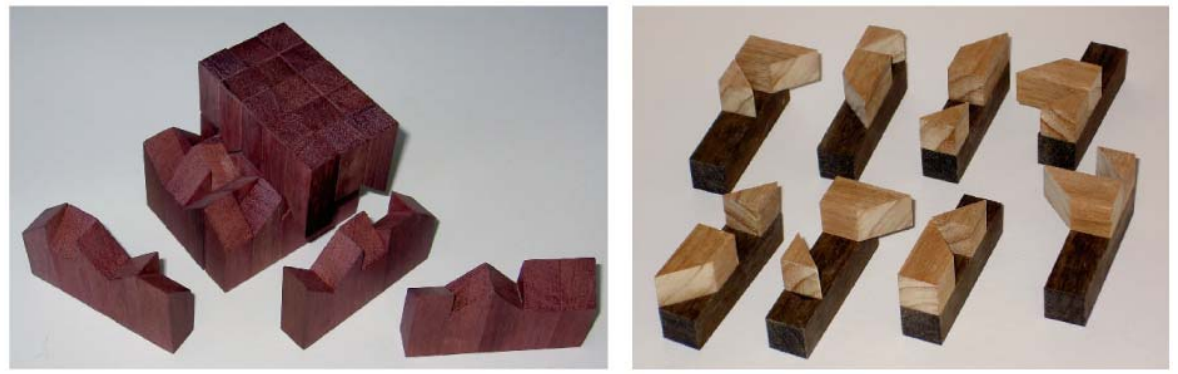

Fig. 2 Examples of two-layer puzzles
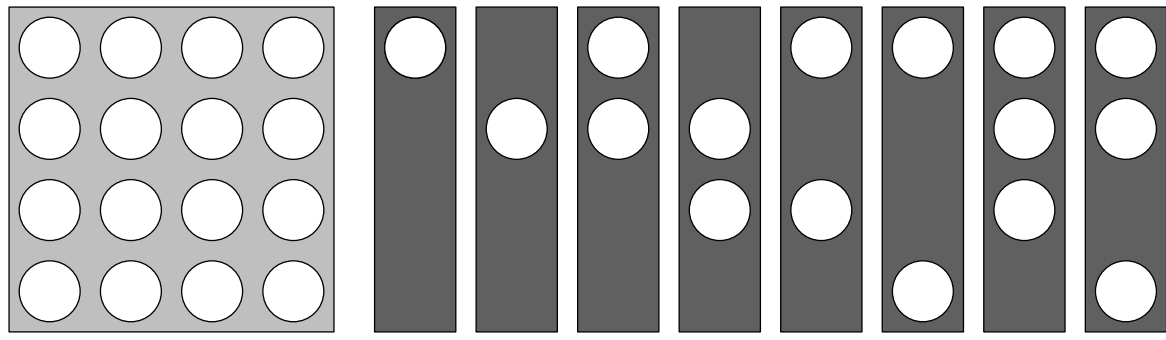

Fig. 3 The 16-holes puzzle by Wim Zwaan 
Partition Puzzles Partition puzzles are puzzles that are based on the well-known PARTITION problem: Given a set of positive integers $v_{1}, \ldots, v_{n}$, partition them in two subsets of equal total value. This problem is NP-complete [7]. The easiest realization as a geometric puzzle is to consider each integer value $v_{i}$ as a $1 \times 1 \times v_{i}$ block and the puzzle is to pack the blocks in a (very long) box of dimensions $1 \times 2 \times V / 2$, where $V=\sum_{i=1}^{n} v_{i}$.

Another partition problem that is NP-complete is 3-PARTITION, which involves partitioning a set of $3 n$ positive integers into $n$ sets of three elements each and with the same subset sum. One puzzle that appears to be directly based on 3-PARTITION is Kunio Saeki's Pipes in Pipe, designed for the 18th International Puzzle Party in 1998. It has 21 little cylinders of different lengths that must fit in seven holes of equal length, see Fig. 4.

Obviously, partitioning a set of integers into three or four subsets of the same total sum is also NP-complete. A realization of a partition puzzle that uses three subsets is shown in Fig. 5. In this puzzle, the slant of $\pi / 3$ and the different ways to deal with the corners make it a variation on a 3-partition puzzle.

Not only solving puzzles based on partition problems is difficult, the creation of geometrically good instances of such partition puzzles is also challenging. A good

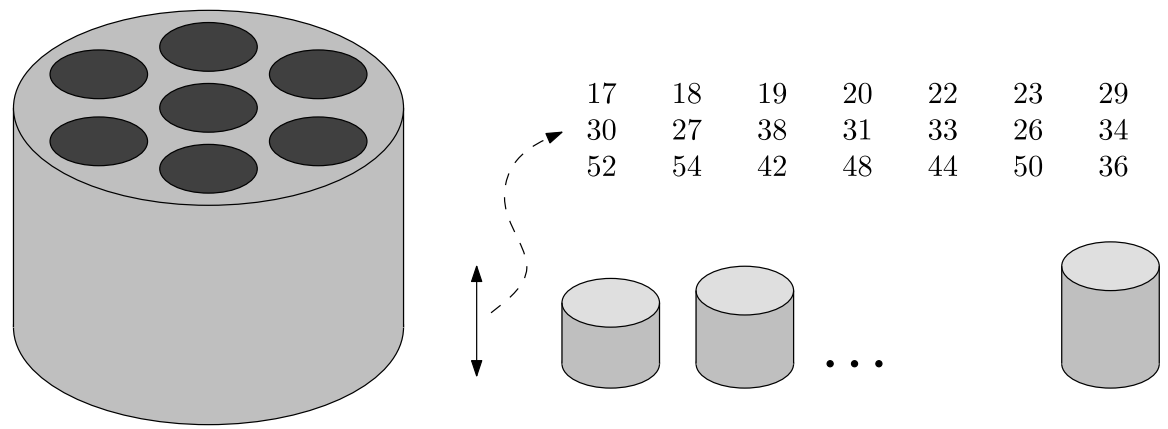

Fig. 4 Partition puzzle by Kunio Saeki

Fig. 5 Partition puzzle based on covering an equilateral triangle with nine pieces of different lengths and shapes

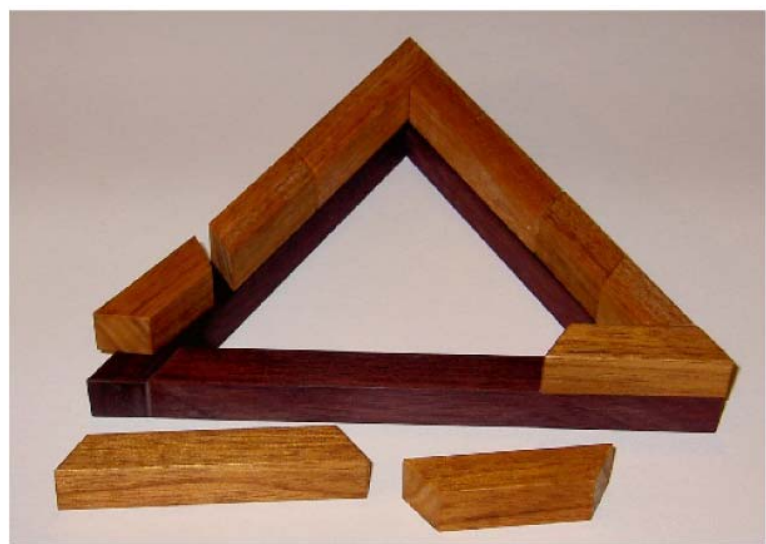


geometric puzzle has the property that it is clear whether a particular solution is the correct solution. Furthermore, it should not be too large, physically. Finally, most good puzzles have only few pieces but are still very hard. The last property can be interpreted for partition puzzles that there should be only one solution. The presence of equal pieces tends to make the solution easier, since it reduces the number of different potential solutions. Therefore, we require that all pieces are distinct. We thus restrict our attention to sets of numbers instead of multisets.

The discussion on clearness of the correct solution can be interpreted as follows: if a set of reals has a solution with two sums of value $V$, then there should not be a small $\varepsilon>0$ such that a different partition into two sets has sums of values $V+\varepsilon$ and $V-\varepsilon$. Here the ratio of $V$ and $\varepsilon$ is important. We will only consider the partition problem for integers. This automatically gives a difference in the subset sums between a correct partition and non-correct partition of 2 . Since a difference of length of $2 \mathrm{~mm}$ is clearly visible, we could take millimeters as units of measurement. But then the sum of a subset that gives a correct solution is the size of the puzzle in millimeters. We would like to find the smallest instance of partition, meaning that the sum of all integers is as small as possible.

We show that for PARTITION, a set of $n$ values exists that has a unique partition into two subsets of equal sum, and of which the sum is $O\left(n^{3}\right)$. Similarly, we show for $k$-subsets partition that a set of $n$ values exist that has a unique partition into $k$ subsets of equal sum, and the sum is $O\left(n^{k+1}\right)$. The proofs are constructive: we give schemes that give instances of the partition problems. In all cases, the $k$ subsets have equal cardinality.

\section{The Complexity of Gate Puzzles}

In this section we show that solving gate puzzles is NP-complete. We consider the simplest form where only two-legged gates occur, and only two heights are used.

Theorem 1 Given a grid of $n \times m$, and $n m / 2$ gates of height 1 or 2 , it is NP-complete to decide if they can be placed on the grid.

Proof Clearly the problem is in NP. To prove NP-hardness we make a reduction from 3-PARTITION, which is NP-complete in the strong sense [7]. An instance of 3 -PARTITION consists of $3 N$ positive integers $v_{1}, \ldots, v_{3 N}$, where each integer is between $B / 4$ and $B / 2$ for some given $B$, and $\sum_{i=1}^{3 N} v_{i}=N B$. The problem is to decide whether a partition of the $3 N$ integers into $N$ subsets exist such that each of these subsets has sum $B$. We transform an integer $v_{i}$ into one gate with leg distance $2 v_{i} N^{2}-1$ and height 2 , and $v_{i} N^{2}-1$ gates of leg distance 1 and height 1 . We ask if all gates fit on a grid of size $2 N^{2} B \times N$, see Fig. 6 .

We first show that gates of height 2 only fit horizontally. It is obvious that they do not fit vertically, but they might fit as the diagonal of a Pythagorean triangle. Note that any gate of height 2 has leg distance $L \geq N^{2}$. It can easily be seen that such a gate cannot fit diagonally, since $L>\sqrt{(L-1)^{2}+(N-1)^{2}}$, see Fig. 7 .

We showed that there are $3 N$ gates that only fit horizontally. There are $N$ rows, and every row will contain three gates of height 2 in any solution. The gates of height 


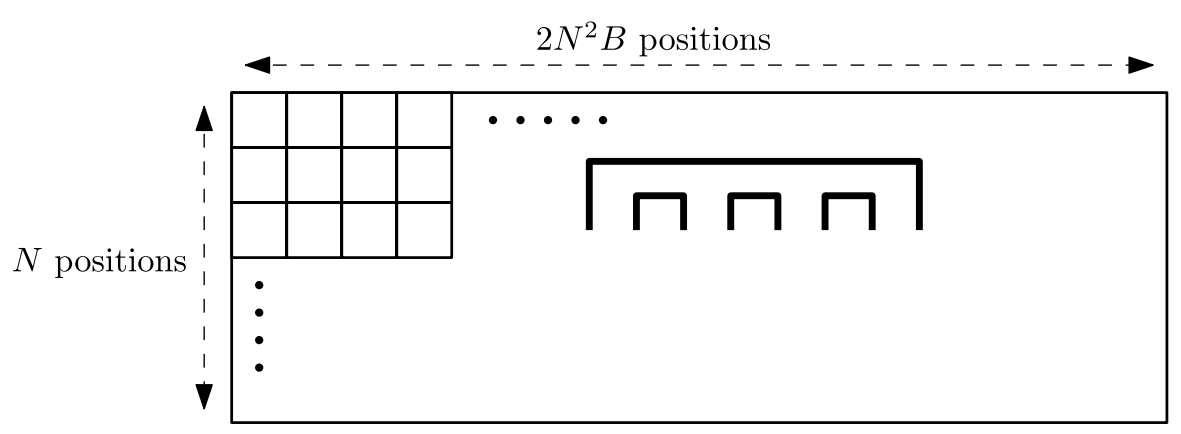

Fig. 6 Reduction of 3-PARTITION to gates

Fig. 7 Since gates have large enough leg distance, they cannot be placed diagonally

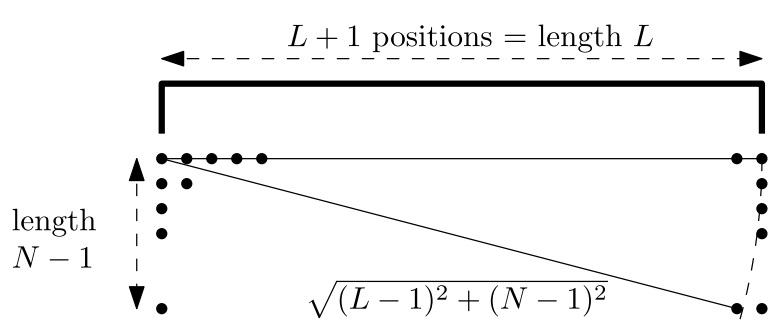

1 are only for making the gate puzzle valid by filling the holes of the whole grid. They fit under the height 2 gate with which they were created.

It is clear that the gate puzzle has a solution if and only if 3-PARTITION has a solution. The reduction is polynomial because 3-PARTITION is NP-complete in the strong sense: even if we write all values in unary notation on the input, the problem is NPcomplete. Therefore, the number of gates obtained after the reduction is polynomial in the input size.

Using gates of heights 1,2 , and 3 , a similar proof can be constructed on a onedimensional grid. We use a single row with $2 N B+2 N$ positions, $N$ gates of height 3 and leg distance $2 B+1$, each integer $v_{i}$ gives rise to a gate of height 2 and leg distance $2 v_{i}-1$, and all other gates have height 1 and leg distance 1 .

\section{The Complexity of Two-Layer Puzzles}

For the NP-completeness proof of two-layer puzzles, we choose a version with $2 n$ pieces of length $n$. Every piece is a row of elements, each of which has height 1 or 2. An example is shown in Fig. 8. We must place $n$ pieces as rows, and the other $n$ pieces upside down as columns on top, such that if a position of the bottom, row layer contains a 1 , then the corresponding position of the top, column layer contains a 2 , and vice versa.

We use the following standard terminology, and an easy observation. A graph is cubic, if each vertex is incident to exactly three edges. A set of edges $M \subseteq E$ is a perfect matching in a graph $G=(V, E)$, if each vertex in $V$ is incident to exactly 
Fig. 8 A two-layer piece with two heights, for a $10 \times 10 \times 3$ block

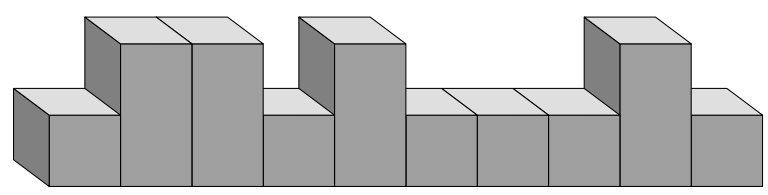

Fig. 9 The horizontal pieces for the reduction; gray is height 2 and white is height 1

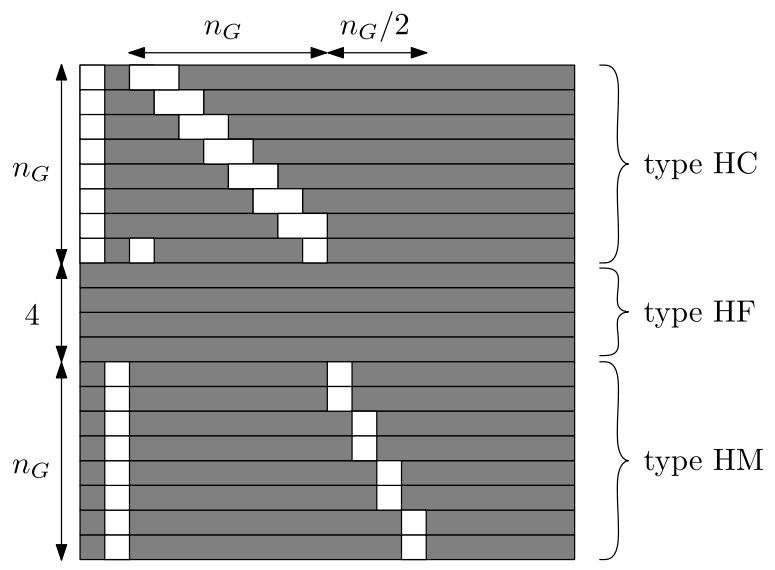

one edge in $M$. We say that a set of edges $C \subseteq E$ forms a Hamiltonian circuit, if it forms a circuit that visits each vertex exactly once. Note that each vertex is incident to exactly two edges in the Hamiltonian circuit. We also denote the Hamiltonian circuit by the sequence in which the vertices are visited.

Lemma 1 Let $G=(V, E)$ be a cubic graph. $G$ has a Hamiltonian circuit, if and only if $E$ can be partitioned into a set of edges that forms a Hamiltonian circuit, and a set of edges that forms a perfect matching in $G$.

Proof The "if" case is trivial. Suppose $G$ has a Hamiltonian circuit. Let $M$ be the edges not on the Hamiltonian circuit. Each vertex $v$ is incident to three edges in $E$, two of which are on the circuit, so one of which belongs to $M$. So $M$ is a perfect matching.

Theorem 2 Given a set of $2 n$ two-layer pieces of length $n$, it is NP-complete to decide if they can be placed to form a solid block of $n \times n \times 3$.

Proof Clearly, the problem is in NP. To prove NP-hardness, we transform from HAMILTONIAN CiRCUiT FOR CUBIC GRAPHS [8].

Let $G=(V, E)$ be a cubic graph, i.e., each vertex in $V$ has exactly three neighbours. Write $n_{G}=|V|$. Note that $n_{G}$ is even, as $G$ is cubic. Without loss of generality, assume that $n_{G} \geq 8$. Assume $V=\left\{v_{1}, \ldots, v_{n_{G}}\right\}$.

We build a collection of $4 n_{G}+8$ two-layer pieces of length $n=2 n_{G}+4$, and distinguish certain types. The main ones are the H-type and V-type, see Figs. 9 and 10 . The construction ensures that all $\mathrm{H}$-type vertices are placed horizontally and 


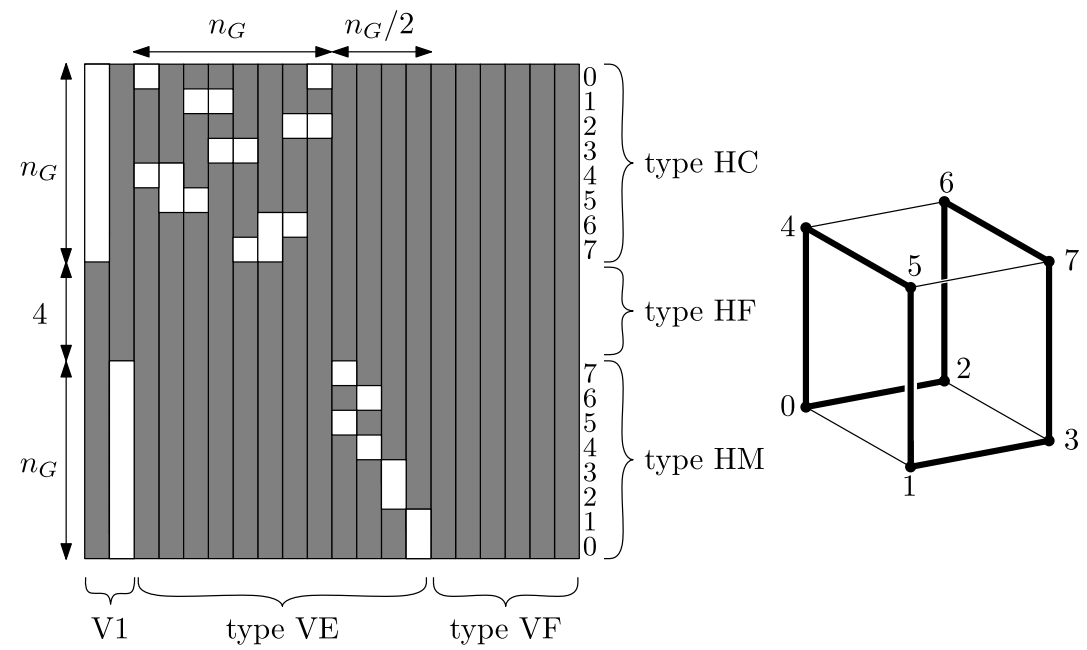

Fig. 10 The vertical pieces for the reduction, showing a solution for the graph of the cube (on the right, with the Hamiltonian circuit highlighted). This time, gray is height 1 and white is height 2, complementing the horizontal pieces of Fig. 9 after permuting them within the groups HC and HM. The vertices of the cube are numbered $0-7$. Two vertices are adjacent if their difference is a power of 2

all V-type pieces are placed vertically, or all H-type pieces are placed vertically, and all V-type pieces are placed horizontally. As we can turn a solution 90 degrees, we assume without loss the former.

The H-type and the V-type have subtypes. We have the following pieces per subtype:

- Type HF: four pieces with all positions at height 2. HF stands for Horizontal, Full.

- Type HC: $n_{G}$ pieces with all but three positions at height 2 . For $1 \leq i \leq n_{G}-1$, we have a piece with positions $1, i+2$, and $i+3$ at height 1 and all other positions at height 2 . We also have a piece with positions 1,3 , and $2+n_{G}$ at height 1 , and all other positions at height 2. HC stands for Horizontal, Circuit: this pieces will be used to model the Hamiltonian circuit. The last piece models the edge that closes the circuit.

- Type HM: $n_{G}$ pieces with all but two positions at height 2 . For $1 \leq i \leq n_{G} / 2$, we have two pieces with positions 2 and $2+n_{G}+i$ at height 1 , and all other positions at height 2. HM stands for Horizontal, Matching: these pieces model a matching in $G$, see Lemma 1.

- Type V1: two pieces with positions 1 until $n_{G}$ (inclusive) at height 2 , and all other positions at height 1.

- Type VE: one piece for each of the $3 n_{G} / 2$ edges in $E$. If $\left\{v_{i}, v_{j}\right\} \in E$, then we take a piece with positions $i$ and $j$ at height 2, and all other positions at height 1 . VE stands for Vertical, Edge, as these pieces model the edges of $G$. (Vertical, Edge, as these model the edges of $G$.)

- Type VF: $n_{G} / 2+2$ pieces with all positions at height 1 . VF stands for Vertical, Full. 
Claim The given collection of pieces has a solution if and only if $G$ has a Hamiltonian circuit.

The claim above shows the correctness, and together with the fact that the collection of pieces can be constructed in polynomial time, given $G$, this will show the NP-hardness. The remainder of the proof of the theorem consists of the proof of the claim.

Suppose $v_{j_{1}}, v_{j_{2}}, \ldots, v_{j_{n_{G}}}$ is a Hamiltonian circuit in $G$. Let $M$ be the set of edges in $G$ that do not belong to the circuit, i.e., let $M$ be the matching as given by Lemma 1 . We place the pieces as follows, see Fig. 10.

Pieces of H-type will always be placed horizontally, pieces of V-type vertically. If we do not state that a piece is reversed, it is placed like its description above.

- One piece of type V1 is placed in the first column.

- The second piece of type V1 is placed in the second column, but reversed, that is, the height 2 squares are at the intersection with rows $n_{G}+5$ until $2 n_{G}+4$.

- For $1 \leq i<n_{G}$, the VE-piece which models the edge $\left\{v_{j_{i}}, v_{j_{i+1}}\right\}$ is placed in column $i+2$.

- The VE-piece that models the edge $\left\{v_{j_{n_{G}}}, v_{j_{1}}\right\}$ is placed in column $n_{G}+2$.

- The $n_{G} / 2$ VE-pieces that model the edges in $M$ are placed reversed in some arbitrary order in the columns $n_{G}+3, \ldots, 3 n_{G} / 2+2$.

- The VF-pieces are placed in columns $3 n_{G} / 2+3, \ldots, 2 n_{G}+4$.

- The HF pieces are placed in rows $n_{G}+1, n_{G}+2, n_{G}+3, n_{G}+4$.

- The HC-piece with height 1 positions at $1, i+2, i+3$ is placed in row $j_{i}$. Note that it fits with the vertical pieces: a vertical piece that has a height 2 position in row $j_{i}$ is either the V1 piece in column 1, or one of the two non-reversed VE-pieces that model the edges $\left\{v_{j_{i-1}}, v_{j_{i}}\right\}$ and $\left\{v_{j_{i}}, v_{j_{i+1}}\right\}$. In each case, the vertical piece has a height 2 position, if and only if the horizontal piece has a height 1 position at the intersection point.

- The HC-piece with height 1 positions at 1,3 , and $2+n_{G}$ is placed in row $j_{n_{G}}$. The analysis that this fits with the horizontal pieces is similar to the previous case, but now we 'wrap around' at $n$.

- For each $i, 1 \leq i \leq n_{G} / 2$ : consider the edge $\left\{v_{k_{1}}, v_{k_{2}}\right\} \in M$ whose VE-piece is placed reversed in column $n_{G}+2+i$. The two HM-pieces with height 1 at positions 2 and $n_{G}+2+i$ are placed in rows $2 n_{G}+4-k_{1}$ and $2 n_{G}+4-k_{2}$. Again, a simple case analysis shows that the piece has height 1 at a location, if and only if at this location, the corresponding horizontal piece has height two. We use here that $M$ is a perfect matching, and thus we use each HM-piece exactly once in this step.

One can verify that we indeed have a solution for the puzzle.

Suppose the collection of pieces has a solution. Consider an arbitrary piece of type HF. Without loss of generality, suppose it is placed horizontally. Then, all four pieces of type HF must be placed horizontally, otherwise we would have a mismatch at the position where the pieces intersect. Each piece of type HC and HM has at most three positions of height 1 , so it cannot be placed vertically. (Otherwise, it would share a position with each of the four HF-type pieces, and at least one of these positions it 
would also have height 2.) As all $2 n_{G}+4$ pieces of H-type are placed horizontally, all pieces of V-type are placed vertically.

V1-type pieces have $n_{G}$ positions of height 2. So, if a V1-type piece is placed in column $i$, then there are $n_{G} \mathrm{H}$-type pieces with height 1 at position $i$ or $2 n_{G}+5-i$. For each $i \in\left\{3, \ldots, 2 n_{G}+2\right\}$, there are at most six H-type pieces with height 1 at positions $i$ or $2 n_{G}+5-i$. There are $n_{G} \mathrm{H}$-type pieces with height 1 at position 1 , and $n_{G} \mathrm{H}$-type pieces with height 1 at position 2 . Thus, one V1-type piece must be placed in column 1 or $2 n_{G}+4$, and one V1-type piece must be placed in column 2 or $2 n_{G}+3$.

Without loss of generality, we suppose one V1-type piece is placed in column 1, and it is not reversed. Consider the H-type pieces at rows $1, \ldots, n_{G}$. At their first position, they meet the height 2 position of the V1-type piece, so they must have height 1 at their first position, and hence be a HC-type piece. Also, their orientation cannot be reversed.

For $1 \leq i<n_{G}$, if the HC-piece with height 1 positions at $1, i+2, i+3$ is in row $j$, set $v_{j_{i}}=j$. Similarly, if the piece with height 1 positions at $1,3,2+n_{G}$ is in row $j$, set $v_{j_{n_{G}}}=j$. This gives a Hamiltonian Circuit. Consider a pair of successive vertices $v_{j_{i}}, v_{j_{i+1}}$. Note that the HC-type pieces in rows $j_{i}$ and $j_{i+1}$ have height 1 at their position $i+3$. So the $\mathrm{V}$-type piece in column $i+3$ must have height 2 at positions $j_{i}$ and $j_{i+1}$. It cannot be a V1-type piece, see above. So, we have a VE-type piece with height 2 at positions $j_{i}$ and $j_{i+1}$, and hence $\left\{v_{j_{i}}, v_{j_{i+1}}\right\} \in E$. A similar argument shows that $\left\{v_{j_{n_{G}}}, v_{j_{1}}\right\} \in E$, and hence we have a Hamiltonian circuit. The finishes the proof the claim, and hence of the theorem.

\section{Designing Partition Puzzles}

In this section we consider partition problems for integers. From the introduction we know that we are mostly interested in instances that are uniquely solvable and have a small total value. We will concentrate on instances of $2 n$, or more generally, $k n$ integers that have a unique partition into 2 subsets (or $k$ subsets, respectively). Moreover, we want all subsets of the partition to have the same cardinality $n$. It is easy to adapt the instances to subsets of different cardinalities, by simply combining pieces.

Lemma 2 Let $S$ be a set of integers that has a partition into two subsets $S_{1}$ and $S_{2}$, each of total value $\frac{1}{2} \cdot \sum_{v \in S} v$. Then this is the unique partition with this property, if and only if no proper subset of $S_{1}$ has equal value to any proper subset of $S_{2}$.

Proof If two such subsets exist, they can be exchanged and the partition is not unique. Conversely, let $S_{1}^{\prime}, S_{2}^{\prime}$ be a different partition with equal sums. Since $S_{1}^{\prime}=\left(S_{1} \backslash\left(S_{1}-\right.\right.$ $\left.\left.\left.S_{1}^{\prime}\right)\right) \cup\left(S_{1}^{\prime}-S_{1}\right)\right)$, the sets $S_{1}-S_{1}^{\prime}$ and $S_{1}^{\prime}-S_{1}$ are nonempty proper subsets of $S_{1}$ and of $S_{2}$ with equal sums.

For $k \geq 3$, the condition of Lemma 2 applied to pairs of sets, is not sufficient to guarantee uniqueness. For example, no two proper subsets of $S_{1}=\{8,14,78\}$, 
$S_{2}=\{9,15,76\}$, or $S_{3}=\{10,13,77\}$ have equal sums, but $S_{1}^{\prime}=\{9,13,78\}, S_{2}^{\prime}=$ $\{10,14,76\}, S_{3}^{\prime}=\{8,15,77\}$ is a different solution.

We present schemes that generate instances of partition with a set $S$ of $2 n$ or $k n$ integers that have a strongly unique solution and a polynomial bound on $\sum_{v_{i} \in S} v_{i}$.

A Simple Scheme for Partition Suppose we wish to generate a set $S$ of $2 n$ integers that has a unique partition into two subsets $S_{1}$ and $S_{2}$ of cardinality $n$ each.

$$
\begin{aligned}
& S_{1}=\{1,2, \ldots, n-1, N\} \\
& S_{2}=\left\{\frac{1}{2} n(n-1)+1, \frac{1}{2} n(n-1)+2, \ldots, \frac{1}{2} n(n-1)+n-1, \frac{1}{2} n(n-1)+n\right\}
\end{aligned}
$$

where $N=\sum_{i=1}^{n}\left(\frac{1}{2} n(n-1)+i\right)-\frac{1}{2} n(n-1)$. Since all integers in $S_{2}$ are larger than the sum of the smallest $n-1$ integers in $S_{1}$, and the $n$-th integer from $S_{1}$ is larger than all integers from $S_{2}$, no proper subset sum from $S_{1}$ can be equal to any proper subset sum of $S_{2}$.

Theorem 3 For any $n \geq 2$, an instance of PARTITION exists with $2 n$ values which has a unique solution in two subsets. Both subsets have $n$ integers, and the subset sum is $\left(n^{3}+n\right) / 2$.

The obvious lower bound corresponding to the theorem is $\sum_{i=1}^{2 n} i=\Omega\left(n^{2}\right)$. From Theorem 3, we have an upper bound of $O\left(n^{3}\right)$ for the subset sum.

A Simple Scheme for Partition into $k$ Subsets The scheme for partition can easily be extended to a scheme for partition into $k$ subsets. The scheme gives subset sums that are polynomial in $n$, but exponential in $k$. We write $V_{i}$ for the sum of the smallest $n-1$ elements in $S_{i}$.

$$
\begin{aligned}
S_{1} & =\left\{1,2, \ldots, n-1, N_{1}\right\} \\
S_{2} & =\left\{V_{1}+1, V_{1}+2, \ldots, V_{1}+n-1, N_{2}\right\} \\
S_{3} & =\left\{V_{2}+1, V_{2}+2, \ldots, V_{2}+n-1, N_{3}\right\} \\
\ldots & \\
S_{k-1} & =\left\{V_{k-2}+1, V_{k-2}+2, \ldots, V_{k-2}+n-1, N_{k-1}\right\} \\
S_{k} & =\left\{V_{k-1}+1, V_{k-1}+2, \ldots, V_{k-1}+n-1, V_{k-1}+n\right\}
\end{aligned}
$$

The integers $N_{1}, \ldots, N_{k-1}$ are chosen so that all subsets have the same subset sum as $S_{k}$. As before we can argue that $N_{1}$ is such that only $1, \ldots, n-1$ are small enough to be with $N_{1}$ and give the right subset sum. Since this fixes $S_{1}$, we can repeat the argument by observing that $N_{2}$ is such that of the remaining integers, only $V_{1}+$ $1, \ldots, V_{1}+n-1$ are small enough to be with $S_{2}$ and give the right subset sum.

Theorem 4 For any $k \geq 2$ and $n \geq 2$, an instance of PARTITION INTO $k$ SUBSETS exists with $k n$ values which has a unique solution in $k$ subsets. All subsets have $n$ integers, and the subset sum is $\Theta\left(n^{k+1}\right)$, if $k$ is fixed. 
Although we presented a scheme that gives uniquely solvable instances of partition of cubic size, the scheme is not satisfactory from the puzzle point of view. It contains an integer that is so large that it is clear which other integers should go in the same subset (which was the argument for uniqueness). So in this case, uniqueness of solution does not imply that a puzzle using this scheme will be difficult. Therefore, we will present another partition scheme and its extension for $k$ subsets that does not have this problem. We will bound the value of the largest integer in the partition problem while obtaining the same bound on the subset sum.

An Improved Scheme for Partition To obtain a scheme that does not have the disadvantage of the simple scheme, choose two sets of integers $1,2, \ldots, n$ and $1,2, \ldots, n-1, n+1$. Multiply each integer in the first set by $n$. Multiply each integer in the second set by $n$ and subtract 1 . This way we get $S_{1}$ and $S_{2}$ :

$$
\begin{aligned}
& S_{1}=\left\{n, 2 n, 3 n, \ldots,(n-1) n, n^{2}\right\} \\
& S_{2}=\left\{n-1,2 n-1,3 n-1, \ldots,(n-1) n-1, n^{2}+n-1\right\}
\end{aligned}
$$

Every subset sum from $S_{1}$ is a multiple of $n$. No proper subset sum from $S_{2}$ is a multiple of $n$, because each integer is $\equiv-1 \bmod n$. Hence, no proper subset sum of $S_{1}$ can be equal to any proper subset sum of $S_{2}$. The sum of all integers in $S_{1}$ is equal to the sum of all integers in $S_{2}$, and is equal to $\frac{1}{2} n(n+1) \cdot n=\left(n^{3}+n^{2}\right) / 2$.

Theorem 5 For any $n \geq 2$, an instance of PARTITION exists with $2 n$ values which has a unique solution in two subsets. Both subsets have $n$ integers, all integers have value $\Omega(n)$ and $O\left(n^{2}\right)$, and the subset sum is $\left(n^{3}+n^{2}\right) / 2$.

Again, $\sum_{i=1}^{2 n} i=\Omega\left(n^{2}\right)$ is a lower bound for the subset sum of Theorem 5, and $O\left(n^{3}\right)$ is an upper bound.

It is easy to adapt the scheme to yield a partition in subsets of different cardinalities: we let $n$ be the desired cardinality of the larger subset in the scheme, and generate $S_{1}$ and $S_{2}$. Then we add $n-m+1$ values in $S_{2}$ to get any cardinality $m$ for the smaller subset, and the partition itself remains unique.

For small values of $n$, we have computed the uniquely solvable instances of PARTITION with smallest subset sum with the help of a computer, by an enumeration algorithm. The instances in the following table turned out to be the unique instances with the given sums, where the two subsets have equal cardinality. (For $n=7$ there are two different smallest instances.) For decompositions into parts of distinct cardinalities, there are smaller solutions. For example, $\{1,3,4,5,6,7\} \cup\{2,24\}$ is the unique solution for an 8 -element set, with sums 26 , but clearly, this leads to a very easy puzzle.

One can see in the table that the constructions of Theorems 3 and 5 are not far from the optimum. Also, the instances for $n=5, n=6$, and $n=7$ share certain characteristics with the construction of Theorem 5: they contain arithmetic progressions, which tends to reduce the number of different subset sums that can be built from a given set. The two solutions for $n=7$ seem to be based on arithmetic progressions with step lengths 7 and 6, respectively. 


\begin{tabular}{cccccc}
\hline$n$ & $S_{1}$ & $S_{2}$ & $\begin{array}{l}\text { Minimum } \\
\text { subset } \\
\text { sum }\end{array}$ & $\begin{array}{l}\text { Simple } \\
\text { scheme } \\
\frac{1}{2}\left(n^{3}+n\right)\end{array}$ & $\begin{array}{l}\text { Improved } \\
\text { scheme } \\
\frac{1}{2}\left(n^{3}+n^{2}\right)\end{array}$ \\
\hline 2 & 1,4 & 2,3 & 5 & 5 & 6 \\
3 & $1,3,9$ & $2,5,6$ & 13 & 15 & 18 \\
4 & $2,7,10,12$ & $3,5,8,15$ & 31 & 34 & 40 \\
5 & $2,7,12,17,22$ & $3,5,10,15,27$ & 60 & 65 & 75 \\
6 & $3,7,10,21,28,35$ & $4,11,14,18,25,32$ & 104 & 111 & 126 \\
7 & $2,9,16,23,30,37,44$ & $5,7,14,21,28,35,51$ & 161 & 175 & 196 \\
7 & $5,11,17,23,29,35,41$ & $1,6,12,18,24,47,53$ & 161 & 175 & 196 \\
\hline
\end{tabular}

An Improved Scheme for Partition into $k$ Subsets We now present a scheme to generate instances of unique partition into 3 subsets, of $n$ integers each, and bounded integers. Below we will generalize it to larger values of $k$. Because we wish to avoid large integers in the instance, we cannot use the inductive argument that was used in the simple scheme for partition into $k$ subsets to obtain uniqueness. Instead, we use the following property to guarantee uniqueness.

Strong Uniqueness. If the total sum of the set $S$ is $k N$, there are only $k$ subsets of $S$ whose sum is $N$.

Choose two integers $p=n$ and $q=n+1$, and let $r=p \cdot q$. The sets of integers in $S_{1}, S_{2}$, and $S_{3}$ are:

$$
\begin{aligned}
& S_{1}=\{r+q, 2 r+q, \ldots,(n-1) r+q, n r+q\} \\
& S_{2}=\{r+p, 2 r+p, \ldots,(n-1) r+p, n r+2 p\} \\
& S_{3}=\{r, 2 r, \ldots,(n-1) r, n r+r\}
\end{aligned}
$$

It is easy to see that the three subset sums are the same. Also, $\sum_{v \in S_{3}} v=\left(\frac{1}{2} n(n+\right.$ $1)+1) p q=\left(\frac{1}{2} n(n+1)+1\right) n(n+1)=\Theta\left(n^{4}\right)$.

To prove uniqueness of the partition, we show strong uniqueness: $S_{1}, S_{2}$, and $S_{3}$ are the only subsets of $S=S_{1} \cup S_{2} \cup S_{3}$, with subset sum $\left(\frac{1}{2} n(n+1)+1\right) r=$ $\frac{1}{3} \cdot \sum_{v_{i} \in S} v_{i}$. Let $S^{\prime}$ be any subset $S$, and let $S^{\prime}$ have $h$ elements from $S_{1}, i$ elements from $S_{2}$, and $j$ elements from $S_{3}$, where $0 \leq h, i, j \leq n$. Since elements from $S_{1}$ are $\equiv q \bmod r$, and $p$ and $q$ are relatively prime, $h>0$ implies that $h=n$ to obtain a total sum of $S^{\prime}$ that is $\equiv 0 \bmod r$. This subset is already $S_{1}$, and $i$ and $j$ have to be 0 , otherwise the total sum is too large. Similarly, $i>0$ implies that $S^{\prime}$ must contain all elements of $S_{2}$ to be $\equiv 0 \bmod r$, and $h=0$ and $j=0$. Finally, if $h=0$ and $i=0$, we need $j=n$ to get a subset $S^{\prime}$ of large enough total sum. Hence, $S_{1}, S_{2}$, and $S_{3}$ are the only subsets of $S$ with sum $\left(\frac{1}{2} n(n+1)+1\right) r$.

To extend this scheme to $k$ sets we need $k-1$ integers $p_{1}, \ldots, p_{k-1}$ that are at least $n$ and pairwise relatively prime. One way to construct such integers is as follows: Let $K$ be the least common multiple of $1,2, \ldots, k-2$. Select $p_{1}$ in the interval $n \leq p_{1}<n+K$, relatively prime to $K$, and set $p_{i+1}=p_{i}+K$, for $i=1, \ldots, k-2$. This yields integers $p_{i}<n+(k-1)$ ! that are pairwise relatively prime. 
We let $r=\prod_{i=1}^{k-1} p_{i}$, and construct sets based on $r$ and $p_{1}, \ldots, p_{k-1}$ as above. For $i=1, \ldots, k-1$, we define

$$
S_{i}=\left\{r+a_{1} \cdot \frac{r}{p_{i}}, 2 r+a_{2} \cdot \frac{r}{p_{i}}, \ldots, n r+a_{n} \cdot \frac{r}{p_{i}}\right\},
$$

where $\left(a_{1}, a_{2}, \ldots, a_{n}\right)$ is a sequence of small positive integers summing $p_{i}$. (A different sequence $\left(a_{1}, a_{2}, \ldots, a_{n}\right)$ is chosen for every $i$.) As before, the last set is just

$$
S_{k}=\{r, 2 r, \ldots,(n-1) r,(n+1) r\}
$$

The subset sum of each set is $\frac{1}{2} n(n+1) \cdot r+r=\Theta\left(n^{k+1}\right)$.

Theorem 6 For any $k \geq 2$ and $n \geq 2$, an instance of PARTITION INTO $k$ SUBSETS exists with $k n$ values which has a unique solution in $k$ subsets. All subsets have $n$ integers, all integers have value $\Omega\left(n^{k-1}\right)$ and $O\left(n^{k}\right)$, and the subset sum is $\Theta\left(n^{k+1}\right)$, if $k$ is fixed.

The construction of Theorem 6 gives an upper bound of $O\left(n^{k+1}\right)$ for the subset sum. We leave it as an interesting open problem to obtain good non-trivial lower bound for the subset sum.

\section{Conclusions and Open Problems}

We showed that two new types of geometric puzzles-gate puzzles and two-layer puzzles-are NP-complete to solve. For puzzles based on partition, we constructed instances with polynomially bounded values that have unique solutions. The sum of the values relates to the physical size of the geometric puzzle. Uniqueness tends to make a puzzle harder, but we saw that a uniquely solvable puzzle may still be easy (for instance, in the simple scheme for partition). Also the improved scheme has an issue that is undesirable from the puzzle design perspective: the greedy approach (always put the largest remaining piece in the set with smallest sum so far) yields the solution.

The strong uniqueness property for partition in three or more subsets is stronger than necessary for having a unique solution. It also has the effect of making the puzzle easier: if the puzzler finds a subset with the right sum, then this subset is certainly part of the overall solution. So for puzzle design purposes, it is interesting to have instances of partition into three or more subsets that have a unique solution, but many subsets with the right summed value.

It appears to be hard to formalize what properties make an instance of partition good for puzzle design. This is a practical open problem. More theoretically, it would be interesting to improve upon the $O\left(n^{k+1}\right)$ bound on the summed value of instances with a unique solution.

Acknowledgements The authors thank Rudy van Vliet and Shmuel Zaks for useful comments. 
Open Access This article is distributed under the terms of the Creative Commons Attribution Noncommercial License which permits any noncommercial use, distribution, and reproduction in any medium, provided the original author(s) and source are credited.

\section{References}

1. Cubism For Fun website. http://cff.helm.lu/

2. Culberson, J.: SOKOBAN is PSPACE-complete. In: Proceedings in Informatics 4 (Int. Conf. FUN with Algorithms 1998), pp. 65-76 (1999)

3. Demaine, E.D.: Playing games with algorithms: Algorithmic combinatorial game theory. In: Proc. of Math. Found. of Comp. Sci., pp. 18-32 (2001)

4. Demaine, E.D., Demaine, M.L.: Puzzles, art, and magic with algorithms. Theory Comput. Syst. 39(3), 473-481 (2006)

5. Demaine, E.D., Hohenberger, S., Liben-Nowell, D.: Tetris is hard, even to approximate. Technical Report MIT-LCS-TR-865, MIT (2002)

6. Flake, G.W., Baum, E.B.: Rush Hour is PSPACE-complete, or why you should generously tip parking lot attendants. Manuscript (2001)

7. Garey, M.R., Johnson, D.S.: Computers and Intractability: A Guide to the Theory of NPCompleteness. Freeman, New York (1979)

8. Garey, M.R., Johnson, D.S., Tarjan, R.E.: The planar Hamiltonian circuit problem is NP-complete. SIAM J. Comput. 5(4), 704-714 (1976)

9. Ratner, D., Warmuth, M.: Finding a shortest solution for the $N * N$-extension of the 15-puzzle is intractable. J. Symb. Comput. 10, 111-137 (1990)

10. Robertson, E., Munro, I.: NP-completeness, puzzles, and games. Util. Math. 13, 99-116 (1978)

11. van Kreveld, M.: Some tetraform puzzles. Cubism For Fun 68, 12-15 (2005)

12. van Kreveld, M.: Gate puzzles. Cubism For Fun 71, 28-30 (2006) 